\title{
Design-oriented modelling of different quenching solutions in induction plasma synthesis of copper nanoparticles
}

\author{
S. Bianconi • M. Boselli • M. Gherardi • V. Colombo
}

\begin{abstract}
The aim of this paper is to compare the effects of different mechanisms underlying the synthesis of copper nanoparticles using an atmospheric pressure radiofrequency induction thermal plasma. A design oriented modelling approach was used to parametrically investigate trends and impact of different parameters on the synthesis process through a thermo-fluid dynamic model coupled with electromagnetic field equations for describing the plasma behaviour and a moment method for describing nanoparticles nucleation, growth and transport. The effect of radiative losses from $\mathrm{Cu}$ vapour on the precursor evaporation efficiency is highlighted, with occurrence of loading effect even with low precursor feed rate due to the decrease in plasma temperature. A method to model nanoparticle deposition on a porous wall is proposed, in which a sticking coefficient is employed to model particle sticking on the porous wall used to carry a quench gas flow into the chamber. Two different reaction chamber designs combined with different quench gas injection strategies (injection through a porous wall for "active" quenching; injection of a shroud gas for "passive" quenching) are analysed in terms of process yield and size distribution of the synthetized nanoparticles. Conclusion can be drawn on the characteristics of each quenching strategy in terms of throughput and mean diameter of the synthesized nanoparticles.
\end{abstract}

\section{Introduction}

The ever-increasing demand for high-quality nanosized materials coming from many industrial and research fields has been spurring the research on viable methods for their synthesis. In particular, process upscaling and cost-effectiveness represent the main challenges, as only a few techniques offer a combination of high throughput and fine tuning of the particle properties. Radio-frequency induction thermal plasmas (RF-ITP) have proved to be effective tools for continuous production of nanoparticles at high throughput, thanks to their distinctive features such as high energy density, high chemical reactivity, high process purity, large plasma volume and long residence time. Furthermore, the large 
number of process variables, such as power, frequency, process gases, phase of the precursor and system geometry, increases the versatility of the process, while the high cooling rate $\left(10^{4}-10^{5} \mathrm{~K} \mathrm{~s}^{-1}\right)$ in the tail of the plasma allows to produce tailored nanoparticles with a narrow particle size distribution (PSD).

Over the last few years, considerable effort was devoted towards the optimization of the parameters of RF-ITP nanoparticle synthesis, in order to improve the affordability and efficiency of the process [1-4]. However, due to the large number of variables that characterize the process, optimization is a challenging task, that can hardly rely on try and fail experimental approaches, because of the equipment cost and the limited amount of information that can be obtained from conventional diagnostic techniques. For this reason the investigation of the performance of nanoparticle synthesis processes in a RF-ITP system has relied extensively on modelling techniques [5-7].

The precursor evaporation efficiency (the fraction of the injected precursor that is effectively evaporated in the plasma) is one of the key parameters to evaluate the performance of the process. It is strongly influenced by precursor dimensions and feed rate: larger particles having higher thermal inertia require longer evaporation times, while high precursor feed rates can cause a local cooling of the plasma in the region where the material is injected, inducing a lowering of the heat flux to the particles [8]. The latter phenomenon, called loading effect, can be considered one of the main reasons limiting the upscaling of the RF-ITP synthesis of nanoparticles to higher throughputs. Moreover, as recently pointed out by Boulos [9], another effect that limits the evaporation efficiency of the injected precursor is the radiative power loss coming from the vapour present in the plasma. This effect is particularly significant for materials with a high emissivity, such as most metal vapours, and results in a strong local cooling of the plasma near the region where the material is injected in the plasma and evaporated, hence decreasing the precursor evaporation efficiency.

Among all the parameters concurring in the process optimization, quench gas injection is perhaps the most thoroughly investigated, due to its strong influence on flow fields, temperature distribution and cooling rates in the system [10-12]. In particular, its main effect is to generate a high cooling rate in the tail of the plasma, allowing for the synthesis of nanoparticles with a narrow PSD. In addition, the quenching configuration (type and position of the injection), together with the geometry of the reaction chamber, has been shown to affect the production rate and the properties of the synthetized nanoparticles [8, 13-14]. Quench gas injection operates with several hundred of standard litres per minute of inert gas, usually argon. Therefore a quenching gas recycling equipment is essential when an industrial application is envisaged, in order to have an economically sustainable process.

In this work, we report on the design-oriented modelling for the optimization of an RF-ITP synthesis process of $\mathrm{Cu}$ nanoparticles starting from a solid precursor. The local cooling of the plasma related to the radiative losses from $\mathrm{Cu}$ vapour present in the torch is reported, highlighting the associated reduction in precursor evaporation efficiency. The effect of the geometry of the reaction chamber and of the quenching configuration is investigated, focusing on process yield and control of the particle deposition on the chamber walls, which should be minimized. The yield of the process is defined as the ratio between the total nanoparticle throughput to the reaction chamber outlet and the precursor feed rate. The adopted simulative model can describe plasma thermo-fluid dynamics, electromagnetic fields, precursor trajectories and thermal history, as well as nanoparticle nucleation and growth. Radiative losses from $\mathrm{Cu}$ vapour and their effect on the precursor evaporation efficiency have also 
been taken into account in the model. Although these results refer to a specific case, the methodologies and considerations can be applied to a broad range of materials and operative conditions.

\section{Modelling approach}

The RF-ITP nanoparticle synthesis process, including plasma thermo-fluid dynamics, electromagnetic field, precursor injection and evaporation, and nanoparticle formation, transport and growth, is modelled within a 2D axisymmetric framework in the ANSYS FLUENT ${ }^{\odot}$ environment [15]. The employed model describes plasma thermo-fluid dynamics, electromagnetic field, precursor behaviour (injection, trajectories, thermal history and evaporation) and nanoparticle formation, transport and growth.

\section{Plasma modelling}

Plasma thermo-fluid dynamics calculations rely on the following assumptions, which have been widely adopted in literature $[5,16]$ :

(i) plasma is in local thermodynamic equilibrium (LTE);

(ii) turbulent effects are taken into account using the standard $\mathrm{k}-\varepsilon$ model;

(iii) plasma is considered optically thin;

(iv) the effect of $\mathrm{Cu}$ vapour on the transport and thermodynamic properties of the gas mixture is neglected, except when accounting for radiative losses;

(v) the viscous dissipation term in the energy equation is neglected;

(vi) displacement currents are neglected.

The governing equations can be written as:

$$
\begin{gathered}
\nabla \cdot \rho \boldsymbol{u}=0 \\
\nabla \cdot(\rho \boldsymbol{u} \boldsymbol{u})=-\nabla p+\nabla \cdot \boldsymbol{\tau}+\rho \boldsymbol{g}+\boldsymbol{F}_{\boldsymbol{L}} \\
\nabla \cdot(\rho \boldsymbol{u} h)=\nabla \cdot\left(\frac{k_{e f f}}{c_{p}} \nabla h\right)+P_{J}-Q_{r}
\end{gathered}
$$

where $\rho$ is the plasma gas density, $\boldsymbol{u}$ is the gas velocity, $p$ is the gas pressure, $\boldsymbol{\tau}$ is the viscous stress tensor, $\boldsymbol{g}$ is the gravitational acceleration, $h$ is the enthalpy of the fluid, $k_{e f f}$ is the effective thermal conductivity that includes both laminar and turbulent contributions, $c_{P}$ is the specific heat at constant pressure and $Q_{r}$ is the volumetric radiative loss. The Lorentz force, $\boldsymbol{F}_{\boldsymbol{L}}$, and Joule dissipation, $P_{J}$, can be expressed as:

$$
\begin{gathered}
\boldsymbol{F}_{\boldsymbol{L}}=\frac{1}{2} \mathfrak{R}\left(\boldsymbol{J} \times \boldsymbol{B}^{*}\right) \\
P_{J}=\frac{1}{2} \mathfrak{R}\left(\boldsymbol{J} \cdot \boldsymbol{E}^{*}\right)
\end{gathered}
$$


where $\boldsymbol{J}$ is the complex phasor for the current density induced in the plasma, $\boldsymbol{B}$ and $\boldsymbol{E}$ are the complex phasors for the magnetic and electric field respectively, and the superscript ' *' indicates the complex conjugate.

The electromagnetic field in the plasma region can be described by Maxwell's equations written in their vector potential formulation:

$$
\nabla^{2} \boldsymbol{A}-i \mu_{0} \sigma \omega \boldsymbol{A}+\mu_{0} \boldsymbol{J}_{\text {coil }}=0
$$

where $\boldsymbol{A}$ is the vector potential complex phasor, $\boldsymbol{J}_{\text {coil }}$ is the complex phasor for the current flowing in the coil, $\mu_{0}$ is the magnetic permeability of free space, $\sigma$ is the plasma electrical conductivity and $\omega=$ $2 \pi f, f$ being the frequency of the electromagnetic field. The electric and magnetic field complex phasors are computed from the vector potential complex phasor using the following expressions: $\boldsymbol{E}=$ $-i \omega \boldsymbol{A}, \boldsymbol{B}=\nabla \times \boldsymbol{A}$. The current induced in the plasma is then obtained from the simplified Ohm's law: $\boldsymbol{J}=\sigma \boldsymbol{E}$.

In calculating the radiative losses, the contributions from both $\mathrm{Ar}$ and $\mathrm{Cu}$ vapour are taken into account, using data from [17]. The volumetric radiative loss $Q_{r}$ was obtained by linear interpolation of the Net Emission Coefficients based the molar fractions of $\mathrm{Ar}$ and $\mathrm{Cu}$ vapour, as suggested by Gleizes et al. [18].

When using the commercial software ANSYS FLUENT ${ }^{\odot}$ to solve fluid equations, the Lorentz forces, ohmic heating and radiative loss terms were implemented in the model using suitable user-defined functions.

Turbulent effects have been taken into account using the standard k- $\varepsilon$ model:

$$
\begin{gathered}
\nabla \cdot(\rho \boldsymbol{u} k)=\nabla \cdot\left(\left(\mu+\frac{\mu_{T}}{\sigma_{k}}\right) \nabla k\right)+G_{k}-\rho \varepsilon \\
\nabla \cdot(\rho \boldsymbol{u} \varepsilon)=\nabla \cdot\left(\left(\mu+\frac{\mu_{T}}{\sigma_{\varepsilon}}\right) \nabla \varepsilon\right)+C_{1 \varepsilon} \frac{\varepsilon}{k} G_{k}-C_{2 \varepsilon} \rho \frac{\varepsilon^{2}}{k}
\end{gathered}
$$

where $k$ is the turbulence kinetic energy related to the root mean square of the velocity fluctuations, $\varepsilon$ is the turbulent dissipation $G_{k}$ is the generation of turbulence kinetic energy due to mean velocity gradients, $C_{1 \varepsilon}, C_{2 \varepsilon}, \sigma_{k}$ and $\sigma_{\varepsilon}$ are constants set to $1.44,1.92,0.25$ and 1.0 respectively. The turbulent viscosity $\mu_{T}$ is calculated as:

$$
\mu_{T}=\rho C_{\mu} \frac{k^{2}}{\varepsilon}
$$

with $C_{\mu}$ constant, set to 0.09 . The standard wall function approach is used to model turbulence in the near-wall region [15]. 
The precursor particles are assumed to be spherical and with a negligible internal resistance to heat transfer. The trajectory of the precursor particles is described by the equation of motion:

$$
\frac{\partial \boldsymbol{u}_{\boldsymbol{P}}}{\partial t}=\left(\frac{3 \rho C_{D}}{4 d_{P} \rho_{P}}\right)\left(\boldsymbol{u}-\boldsymbol{u}_{\boldsymbol{P}}\right)\left|\boldsymbol{u}-\boldsymbol{u}_{\boldsymbol{P}}\right|+\boldsymbol{g}
$$

where $\rho_{P}, \boldsymbol{u}_{\boldsymbol{P}}$ and $d_{P}$ are the precursor particle density, velocity and diameter, respectively, and $C_{D}$ is the drag coefficient, computed as in [19].

The thermal history of the precursor particles is governed by the following energy balance equation:

$$
\frac{d T_{P}}{d t}=\frac{q}{m_{P} c_{P}}
$$

where $T_{P}$ and $m_{P}$ are the temperature and mass of the particle and $c_{P}$ its specific heat. The heat flux to the particle $q$ is obtained by [20]:

$$
q=A_{P} h_{C}\left(T-T_{P}\right)-A_{P} \varepsilon_{P} \sigma_{S B}\left(T_{P}^{4}-T_{\mathrm{a}}^{4}\right)
$$

where $A_{P}$ is the surface area of the particle, $h_{C}$ is the local convective heat transfer coefficient, $\varepsilon_{P}$ is the emissivity of the particle, $\sigma_{S B}$ is the Stefan-Boltzmann constant and $T_{\mathrm{a}}$ is the temperature of the walls of the reaction chamber, i.e. $300 \mathrm{~K}$. When the particle reaches the melting point, the liquid fraction $x_{P}$ is changed according to the following particle heat balance equation:

$$
\frac{d x_{P}}{d t}=\frac{6 q}{d_{P} \rho_{P} \lambda_{m}}
$$

where $\lambda_{m}$ is the latent heat of particle melting. When the particle is fully in the liquid phase, evaporation is modelled assuming that mass transfer starts when the particle reaches the boiling temperature. In this case, the particle heat and mass balance can be written as:

$$
\frac{d d_{P}}{d t}=-\frac{2 q}{\rho_{P} \lambda_{v}}
$$

where $\lambda_{v}$ is the latent heat of vaporization. Finally, the precursor vapour concentration is obtained solving the vapour conservation equation:

$$
\nabla \cdot[u N]=\nabla \cdot\left[D_{\text {vap }}^{\text {tot }} \nabla N\right]+\frac{P}{\rho_{P} v_{0}}-\frac{S}{\rho_{P} v_{0}}
$$

where $N$ is the vapour concentration, $v_{0}$ is the monomer volume, $P$ is the vapour mass source term accounting for the production rate due to evaporation, whereas $S$ is the vapour mass source term accounting for the consumption on behalf of nucleation and condensation, and $D_{v a p}^{\text {tot }}$ is the total vapour diffusion coefficient, accounting for turbulence as described in [6]. 


\section{The nanoparticle model}

The nanoparticle synthesis process can be described by the aerosol general dynamic equation (GDE) [21], which accounts for the nanoparticle nucleation, growth and transport in the surrounding fluid via convection, diffusion and thermophoresis:

$$
\begin{aligned}
\frac{\partial}{\partial t} n\left(v_{P}\right) & +\nabla \cdot\left[\boldsymbol{u} n\left(v_{P}\right)\right] \\
= & \nabla \cdot\left[D_{P}\left(v_{P}\right) \nabla n\left(v_{P}\right)\right]+I \delta_{D}\left(v_{P}-v_{P}^{*}\right)-\frac{\partial}{\partial v_{P}}\left[G n\left(v_{P}\right)\right] \\
& +\frac{1}{2} \int_{0}^{v_{P}} \beta\left(v_{P}^{\prime}, v_{P}-v_{P}^{\prime}\right) n\left(v_{P}^{\prime}\right) n\left(v_{P}-v_{P}^{\prime}\right) d v_{P}^{\prime} \\
& -\int_{0}^{\infty} \beta\left(v_{P}, v_{P}^{\prime}\right) n\left(v_{P}^{\prime}\right) n\left(v_{P}\right) d v_{P}^{\prime}
\end{aligned}
$$

where $v_{P}$ is the particle volume, $n\left(v_{P}\right)$ is the PSD function, $\boldsymbol{u}$ is the fluid velocity, $I$ is the particle nucleation rate, $G$ is the heterogeneous condensation rate, $\delta_{D}$ represents the Dirac delta function, the superscript ' $*$ ' denotes the critical state, $\beta$ is the interpolative collision frequency function as proposed by Fuchs [22], and $D_{P}$ is the particle diffusion coefficient as proposed by Phanse et al. [23].

The moment method. In this model the aerosol GDE is mathematically reformulated by means of the moment method [24] to obtain a system of equations that is easier to solve. The following assumptions are made:

(i) spherical nanoparticles;

(ii) identical nanoparticle temperature to that of the plasma flow;

(iii) heat generation by condensation is neglected;

(iv) negligible electrical charge of nanoparticles in the reaction chamber;

(v) condensing vapour is treated as an ideal gas;

(vi) nanoparticle size with a uni-modal log-normal distribution.

The moment method handles the first three moments of the PSD function, defined as:

$$
M_{k}=\int_{0}^{\infty} v_{P}^{k} n\left(v_{P}\right) d v_{P} \quad k=0,1,2
$$

The zeroth moment represents the local concentration of the generated nanoparticles, while the first moment corresponds to their total volume. The steady-state aerosol GDE is the written in the form:

$$
\nabla \cdot\left[\boldsymbol{u} M_{k}\right]=\nabla \cdot\left[D_{P}^{\text {tot }} \nabla M_{k}\right]+\left[\dot{M}_{k}\right]_{n u c l}+\left[\dot{M}_{k}\right]_{\text {cond }}+\left[\dot{M}_{k}\right]_{\text {coag }} \quad k=0,1,2
$$


where the terms $\left[\dot{M}_{k}\right]$ represent the net production rates due to nucleation, condensation and coagulation, whose exhaustive description can be found in [20]. $D_{P}^{t o t}$ is the total diffusion coefficient accounting for both turbulent and laminar diffusion, calculated as in [6]. The system is then mathematically closed by the definitions of geometric standard deviation $\sigma_{G}$, geometric mean volume $v_{G}$, and the relation between moments:

$$
\begin{gathered}
\ln ^{2} \sigma_{g}=\frac{1}{9} \ln \left(\frac{M_{0} M_{2}}{M_{1}}\right) \\
v_{g}=\frac{M_{1}^{2}}{M_{0}^{3 / 2} M_{2}^{1 / 2}} \\
M_{k}=M_{0} v_{g}^{k} \exp \left(\frac{9}{2} k^{2} \ln \sigma_{g}\right)
\end{gathered}
$$

\section{Computational domain, material properties and boundary conditions}

The computational domain includes an induction plasma torch and a reaction chamber for the synthesis of nanoparticles. The torch geometry and corresponding dimensions are reported in Figure 1a.
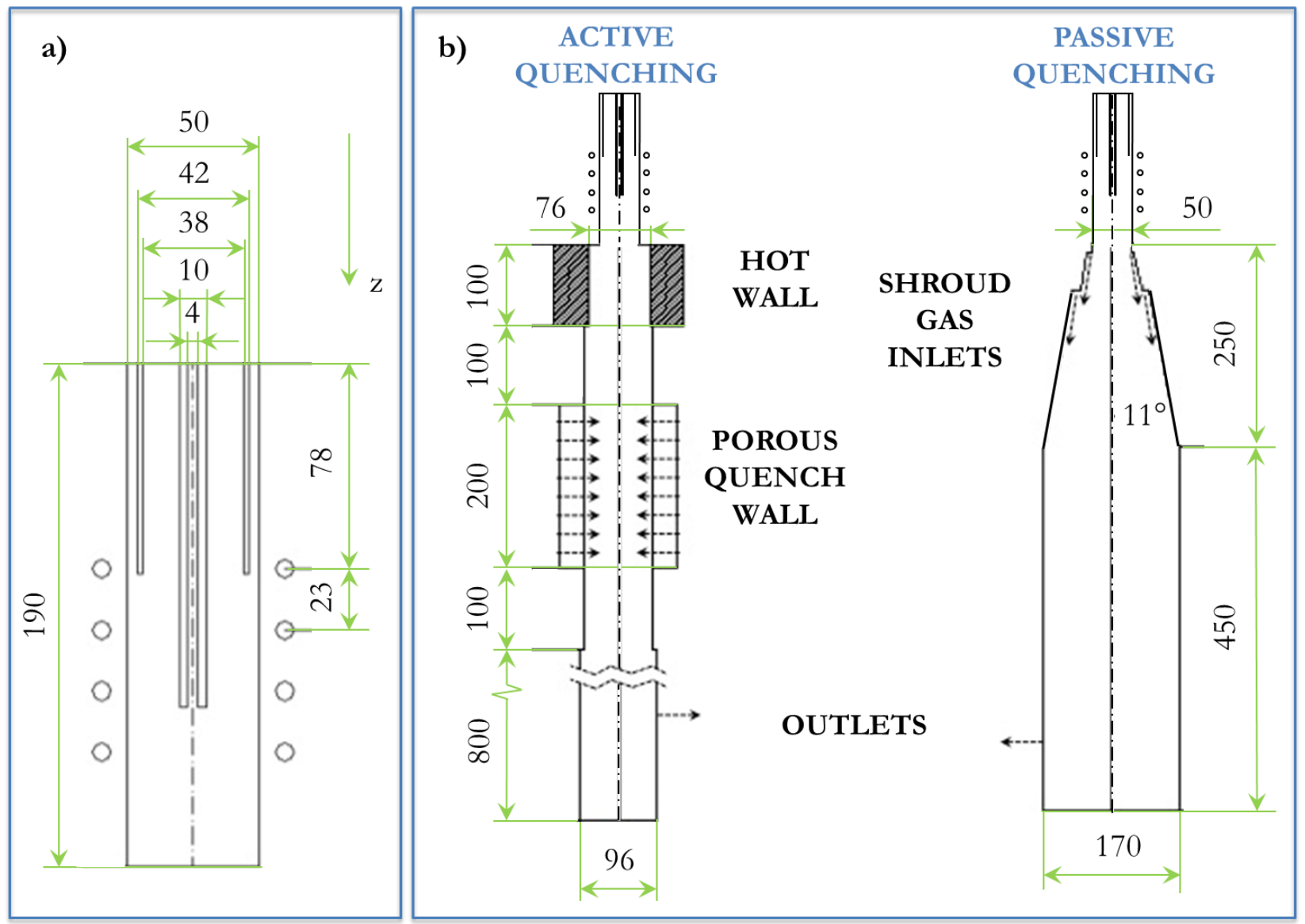

Figure 1 Schematic of the torch (a), and of the reaction chambers (b). Dimensions are in $\mathrm{mm}$. 
The working gas (pure Ar) is supplied through three different inlet regions located in the head of the torch: carrier gas from a central injection probe, central gas from the gap between the probe and the inner confinement tube, and sheath gas from the gap between the inner and outer confinement tubes. The imposed inlet mass flow rates are $4 \mathrm{slpm}$ of carrier gas and $12 \mathrm{slpm}$ of central gas. The sheath gas mass flow rate has been adjusted between $80 \mathrm{slpm}$ and $150 \mathrm{slpm}$ as a function of the different power levels investigated. As usually done for this type of systems when operated at high power, the axial injection is imposed for sheath and carrier gas while the central gas is injected tangentially. Two reaction chamber geometries have been considered: a cylindrical reaction chamber and a reaction chamber composed of a conical top part and a cylindrical bottom part, such as those presented in [13]. There is a thermally insulated wall section at the top of the cylindrical reaction chamber, aimed at increasing the evaporation efficiency, while the conical reaction chamber is directly connected to the plasma torch. Two quenching strategies have been investigated: active and passive quenching. In the former chamber the quench gas is supplied through a porous wall section ("active" quenching), as shown in Fig 1b, while in the latter a shroud gas ("passive" quenching) is injected through two inlet regions located in the upper region of the conical section $\left(\mathrm{Q}_{1}\right.$ and $\mathrm{Q}_{2}$ in Fig 1c). Simulations were carried out for three different plate power levels, $35 \mathrm{~kW}, 60 \mathrm{~kW}$ and $80 \mathrm{~kW}$, corresponding respectively to $25 \mathrm{~kW}, 39 \mathrm{~kW}$ and $50 \mathrm{~kW}$ coupled power to the plasma, assuming a plate coupling efficiency of $65 \%$, as suggested by Boulos for this type of systems [9]. The operating pressure and the coil current frequency are set to $100 \mathrm{kPa}$ and $3.8 \mathrm{MHz}$, respectively. The electromagnetic field equations are solved in a domain extending $95 \mathrm{~mm}$ outside of the torch in the radial direction and $200 \mathrm{~mm}$ upstream the outlet of the torch in the axial direction, using the extended field approach [25]. A no-slip boundary condition is applied on all the internal walls, while a $300 \mathrm{~K}$ temperature is fixed at the external walls of the torch and at the internal walls of the chamber: this boundary condition takes into account that in the 25-50 kW power range water cooling is adopted for the outer confinement tube and for most of the reaction chamber walls. In order to investigate the effects of a thermally insulated wall section at the torch outlet some simulations were also carried out using an adiabatic boundary condition on the wall section located at the top of the cylindrical chamber, as shown in Fig 1 b.

The boundary conditions for turbulence equations at the torch inlet are set according to Chen et al. [26], while a uniform flow with $10 \%$ turbulent intensity was specified for the quench gas inlet through the porous wall [15]. The values of all three moments are set to zero at the inner walls of the reaction chamber, except for the case of the quench gas flowing into the chamber through the porous wall section. This condition describes the assumption that all the nanoparticles that are deposited on the water cooled walls are considered as a loss. Only a fraction of the nanoparticle flux deposits on the porous wall due to the quench gas flowing inwards [16]; in order to model this phenomenon, a sticking coefficient was introduced in the particle balance equation at the wall, investigating its effect with values ranging from $0 \%$ to $100 \%$. The concentration of nanoparticles on the quench wall boundary is then set as a function of the sticking coefficient $\alpha$, according to the particle balance at the porous wall. For instance, referring to the first moment, the following mass balance equation can be written:

$$
(1-\alpha) \varphi=\Gamma
$$


where $\Gamma$ is the convective radial mass flux of nanoparticles due to the quench gas flowing inwards through the porous wall, and $\varphi$ is the nanoparticle diffusive flux to the wall, as described in [13]:

$$
\begin{gathered}
\varphi=\int-\rho_{P} D_{P}^{t o t}\left|\nabla M_{1}\right| d A_{w} \\
\Gamma=\int \rho_{P} \boldsymbol{u} M_{1} d A_{w}
\end{gathered}
$$

$A_{w}$ being the boundary surface area of each computational cell on the porous wall section. The values of the moments at the porous wall section are thus set according to the following expression:

$$
M_{k}{ }^{(i)}=\frac{(1-\alpha) \int-\rho_{\mathrm{P}} \mathrm{D}_{\mathrm{P}}^{\text {tot }}\left|\nabla \mathrm{M}_{\mathrm{k}}\right|^{(i-1)} \mathrm{dA}_{\mathrm{w}}}{\int \rho_{P} \boldsymbol{u} d A_{w}} \quad k=0,1,2
$$

where the superscript $(i)$ and $(i-1)$ refer to the iteration number of the solver. Plasma thermodynamic and transport properties for pure Ar in LTE have been computed as in [27]. The physical properties for $\mathrm{Cu}$ powders are reported in Table 1 and were taken from [28, 29]. Micro-sized copper precursor particles (mean diameter $=7.3 \mu \mathrm{m}$ ) are injected through the probe by means of the carrier gas with different feed rates spanning from $0.1 \mathrm{~g} / \mathrm{s}$ to $1 \mathrm{~g} / \mathrm{s}$.

Table 1: Physical properties for copper powders

\begin{tabular}{lll}
\hline Property & Units & Value \\
\hline$\rho_{p}$ & $\mathrm{~kg} \mathrm{~m}^{-3}$ & 8900 \\
$c_{p}$ & $\mathrm{~J} \mathrm{~kg}^{-1} \mathrm{~K}^{-1}$ & 385 \\
$\lambda_{m}$ & $\mathrm{~J} \mathrm{~kg}^{-1}$ & $2.05 \times 10^{5}$ \\
$\lambda_{v}$ & $\mathrm{~J} \mathrm{~kg}^{-1}$ & $4.74 \times 10^{6}$ \\
$T_{m}$ & $\mathrm{~K}$ & $1357.6-2.55 \times 10^{6} \mathrm{~d}_{\mathrm{p}}^{-1}$ \\
$T_{v}$ & $\mathrm{~K}$ & 2385 \\
$\varepsilon$ sol. & $\mathrm{adm}$ & 0.6 \\
$\varepsilon$ liq. & $\mathrm{adm}$ & 0.3 \\
$k_{p}$ & $\mathrm{~W} \mathrm{~m} \mathrm{~K}^{-1}$ & 320 \\
$M$ & $\mathrm{~g} \mathrm{~mol}^{-1}$ & 63.546 \\
$R_{0}$ & $\mathrm{~nm}$ & 0.135 \\
$\gamma$ & $\mathrm{N} \mathrm{m}^{-1}$ & $1.257-2 \times 10^{-4}(\mathrm{~T}-1356)$ \\
$p_{s a t}$ & $\mathrm{~Pa}$ & $10 \wedge\left(10.855-16415 \mathrm{~T}^{-1}\right)$ \\
\hline
\end{tabular}




\section{Results and discussion}

Precursor evaporation

The precursor evaporation rate and evaporation efficiency were calculated for different operating conditions (coupled power and precursor feed rate) and using two different physical models for the energy balance in the plasma (either including or neglecting the radiative losses from $\mathrm{Cu}$ vapour). At first, the case of a cylindrical chamber (Figure 1) without thermally insulated section is presented, assuming all the chamber walls to be water cooled. The evaporation rate $\Omega$ is defined as the amount of $\mathrm{Cu}$ vapour produced per unit time:

$$
\Omega=\int S_{i} d V
$$

where $S_{i}$ is the amount of vapour produced per unit time and unit volume in each computational cell and $V$ is the volume of the torch and chamber regions. The evaporation efficiency is defined as the ratio between the evaporation rate and the precursor feed rate. Figure 2 offers a comparison between the temperature fields and vapour concentration distributions in the torch and cylindrical chamber obtained either including or neglecting the radiative power losses from $\mathrm{Cu}$ vapour in the energy balance equation: a significant cooling of the plasma in the region downstream of the injection probe due to the presence of $\mathrm{Cu}$ vapour is highlighted; for a plate power level of $60 \mathrm{~kW}$ (39 kW coupled power), the total radiative power loss in the torch increases from $16 \mathrm{~kW}$ to $27 \mathrm{~kW}$. These results are in agreement with calculations reported by Boulos [9]. 


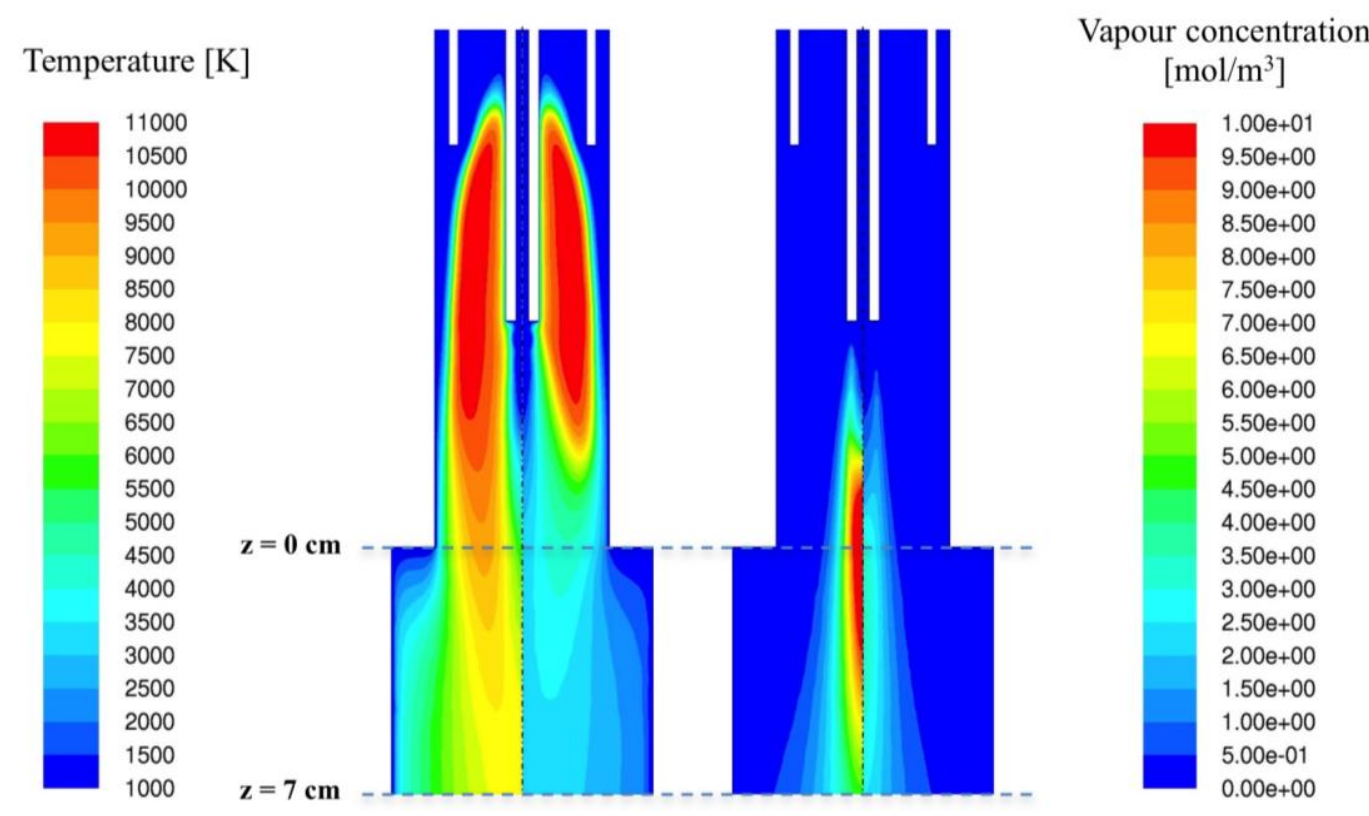

Figure 2 Temperature and vapour concentration profiles in the torch and chamber calculated neglecting the effect of radiative losses from $\mathrm{Cu}$ vapour (left) and considering them (right). Simulations were carried out for the case at $60 \mathrm{~kW}$ plate power ( $39 \mathrm{~kW}$ coupled power) and $0.46 \mathrm{~g} / \mathrm{s}$ precursor feed rate.

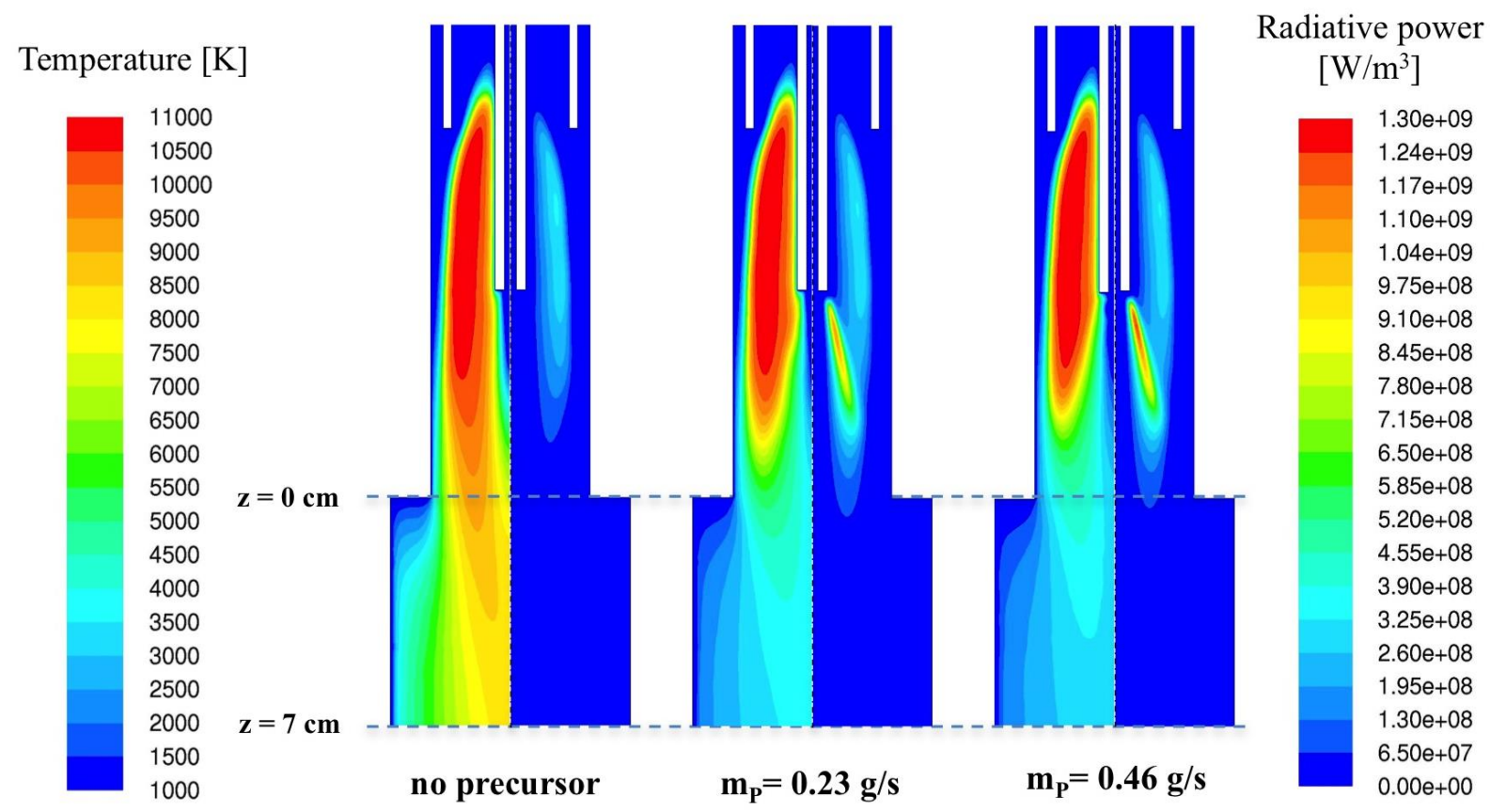

Figure 3 Temperature profile (left) and radiative power losses (right) in the torch and chamber for three different precursor feed rates. Simulations were carried out taking into account the radiative losses from $\mathrm{Cu}$ vapour, for the case at $60 \mathrm{~kW}$ plate power $(39 \mathrm{~kW}$ coupled power).

Figure 3 shows the temperature fields and radiative power loss profiles (including the radiative losses from $\mathrm{Cu}$ vapour) in the torch and cylindrical reaction chamber for three different precursor feed rates. The temperature field for the case without precursor injection in Figure 3 is quite similar to that of the 
case without radiative losses (Figure 2), highlighting that the local cooling effect due to precursor evaporation is negligible compared to the cooling induced by radiative losses from $\mathrm{Cu}$ vapour. The evaporation rates and efficiencies as a function of precursor feed rate are reported in Figure 4 for three different plate power levels $(35,60$ and $80 \mathrm{~kW}$, corresponding to coupled power of 25,39 and $50 \mathrm{~kW}$, respectively). Complete evaporation of the precursor injected (up to $1 \mathrm{~g} / \mathrm{s}$ of feed rate) was obtained for all three power levels in the simulations carried out without taking into account the radiative losses from $\mathrm{Cu}$ vapour (non-realistic conditions). Only in the case of $35 \mathrm{~kW}$ plate power level ( $25 \mathrm{~kW}$ coupled power), the precursor evaporation rate decreases when the precursor feed rate is increased over $0.46 \mathrm{~g} / \mathrm{s}$ as a consequence of the loading effect [8]. Conversely, the evaporation efficiency (Table 2) is significantly lower when including the radiative losses from $\mathrm{Cu}$ vapour, with a more pronounced decrease for higher precursor feed rates.

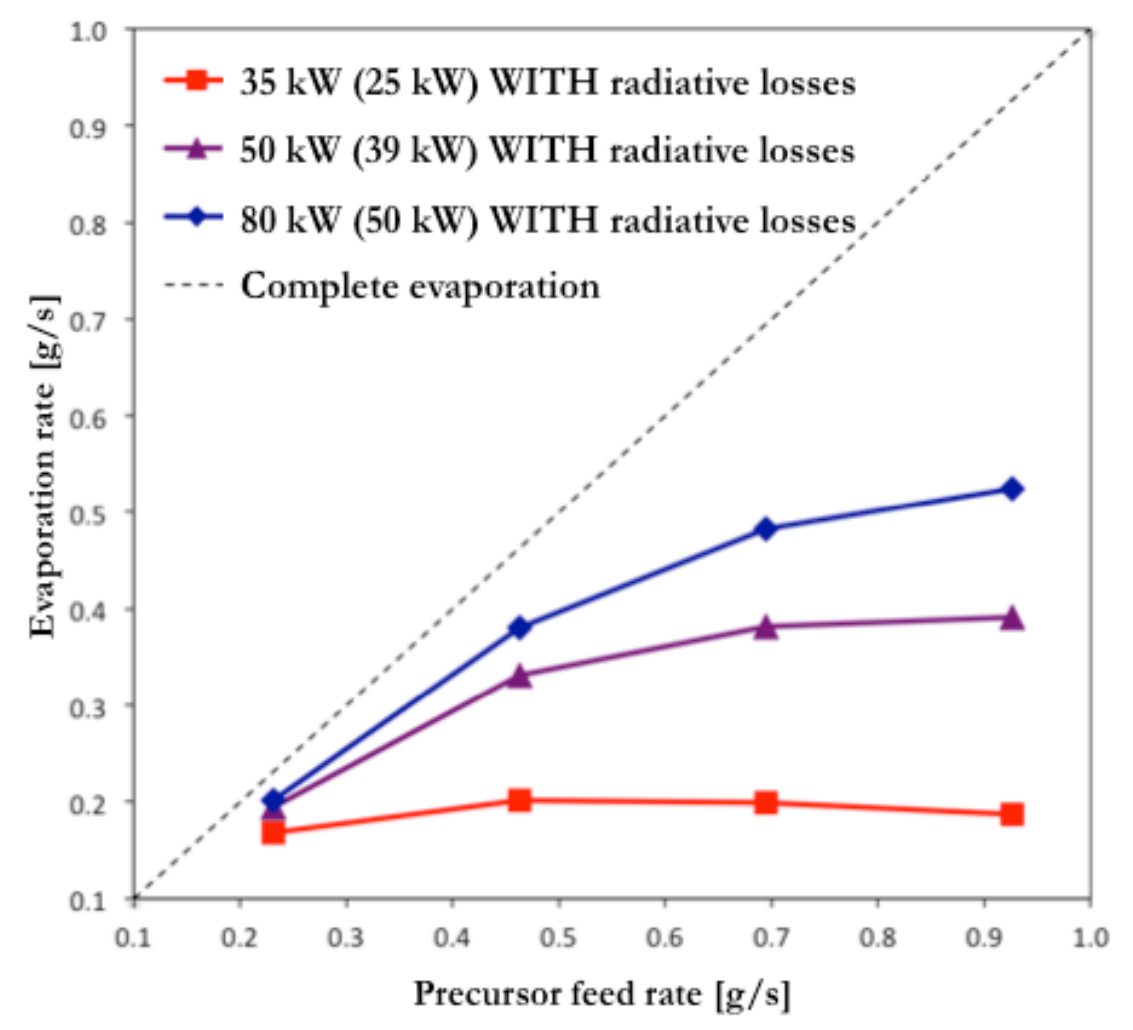

Figure 4 Evaporation rates as a function of precursor feed rate for different plate power levels $(35,60$ and 80 $\mathrm{kW}$, corresponding to 25,39 and $50 \mathrm{~kW}$ coupled power, respectively), taking into account radiative power losses from $\mathrm{Cu}$ vapour. 
Table 2: Evaporation efficiencies as a function of precursor feed rate for three different plate power levels ( 35 , 60 and $80 \mathrm{~kW}$, corresponding to 25,39 and $50 \mathrm{~kW}$ coupled power, respectively), taking into account radiative power losses from $\mathrm{Cu}$ vapour.

\begin{tabular}{ccccc}
\hline $\begin{array}{c}\text { Plate power } \\
\text { (coupled power) }\end{array}$ & 0.23 & 0.46 & 0.69 & 0.93 \\
\hline $35 \mathrm{~kW}(25 \mathrm{~kW})$ & $73 \%$ & $44 \%$ & $29 \%$ & $20 \%$ \\
\hline $60 \mathrm{~kW}(39 \mathrm{~kW})$ & $84 \%$ & $72 \%$ & $55 \%$ & $42 \%$ \\
\hline $80 \mathrm{~kW}(50 \mathrm{~kW})$ & $87 \%$ & $82 \%$ & $69 \%$ & $57 \%$ \\
\hline
\end{tabular}

Higher evaporation efficiency can be achieved adding a thermally insulated wall section at the top of the cylindrical chamber in order to maintain higher temperatures in the region of precursor evaporation, as reported in Table 3 for the case at $60 \mathrm{~kW}$ plate power ( $39 \mathrm{~kW}$ coupled power). This effect is more relevant for higher precursor feed rates: for instance in the case of $0.93 \mathrm{~g} / \mathrm{s}$ precursor feed rate the evaporation efficiency increases from $42 \%$ of the injected precursor to $54 \%$.

Table 3: Evaporation efficiencies as a function of precursor feed rate for the cases with and without a thermally insulated wall section at the top of the chamber, as represented in Figure1. Simulations were carried out taking into account radiative power losses from $\mathrm{Cu}$ vapour and for a $60 \mathrm{~kW}$ plate power level (39 $\mathrm{kW}$ coupled power).

\begin{tabular}{ccccc}
\hline $\begin{array}{c}\text { Thermally insulated } \\
\text { wall section }\end{array}$ & 0.23 & 0.46 & 0.69 & 0.93 \\
\hline No & $84 \%$ & $72 \%$ & $55 \%$ & $42 \%$ \\
\hline Yes & $89 \%$ & $79 \%$ & $67 \%$ & $54 \%$ \\
\hline
\end{tabular}

Nanoparticle synthesis with a cylindrical chamber

The process yield and mean diameter of the synthetized nanoparticles have been calculated for different quench gas flow rates and using different sticking coefficients to model the particle deposition on the porous wall section. The case of a cylindrical chamber (Figure 1) without thermally insulated section is presented, assuming all the chamber walls to be water cooled, since no sensible differences in the nanoparticle synthesis were found from simulations performed assuming a thermally insulated wall. The simulations were performed for the cases at $60 \mathrm{~kW}$ plate power ( $39 \mathrm{~kW}$ coupled power) and 0.46 $\mathrm{g} / \mathrm{s}$ precursor feed rate. The total nanoparticle throughput $\Gamma$ is calculated as the integral of the convective radial mass flux of nanoparticles at the outlet of the reaction chamber:

$$
\Gamma=\int \rho_{P} \boldsymbol{u} M_{1} d A_{\text {outlet }}
$$


where $A_{\text {outlet }}$ is the boundary surface area of each computational cell on the chamber outlet. The mass balance of nanoparticles in the chamber can then be written as:

$$
\Lambda=\Gamma+\sum_{i} \Phi_{i}
$$

where $\Lambda$ is the rate of vapour consumption and $\sum_{i} \Phi_{i}$ is the sum of the nanoparticle deposition fluxes on all the chamber wall sections, computed as the integral of the nanoparticle mass diffusion flux on the chamber walls:

$$
\Phi_{i}=\int \varphi d A_{w_{i}}
$$

where $A_{w_{i}}$ is the boundary surface area of each computational cell on the chamber wall section. The mean diameter of the synthetized nanoparticles at the reaction chamber outlet is computed from the moments of the PSD function as shown by Pratsinis [24]. In all the examined cases it was verified that the $\mathrm{Cu}$ vapour fully condenses before reaching the chamber outlet (i.e. the evaporation rate $\Omega$ corresponds to the rate of vapour consumption $\Lambda$ ). This unexpected result also occurs in operating conditions with no quench gas flow rate, the cooling effect of the $\mathrm{Cu}$ radiation being strong enough to condensate all the vapour, at least in the investigated range of precursor feed rates $(0,23-0,93 \mathrm{~g} / \mathrm{s})$ and coupled power $(25-50 \mathrm{~kW})$. The process yield, defined as the ratio between the total nanoparticle throughput and the precursor feed rate, mean diameter of the synthetized nanoparticles and nanoparticle deposition rate on the porous wall section are reported in Table 4 for three different regimes of quench gas flow of Ar (no quenching, 500 slpm and 1000 slpm flow rate).

Table 4: Summary of the performances of the nanoparticle synthesis process, simulated for the cylindrical chamber using different sticking coefficients $(100 \%, 50 \%$ and $0 \%)$. Simulations were carried out for $60 \mathrm{~kW}$ plate power ( $39 \mathrm{~kW}$ coupled power), precursor feed rate of $0.46 \mathrm{~g} / \mathrm{s}$ and quench gas flow rates of 0 slpm, 500 slpm and 1000 slpm.

\begin{tabular}{llccc}
\hline Quench & $\begin{array}{l}\text { Sticking } \\
\text { coefficient }\end{array}$ & $\begin{array}{l}\text { Particle deposition on } \\
\text { porous wall (\%) }\end{array}$ & $\begin{array}{l}\text { Yield } \\
(\%)\end{array}$ & $\begin{array}{l}\overline{\boldsymbol{d}}_{\boldsymbol{P}} \text { at outlet } \\
(\mathrm{nm})\end{array}$ \\
\hline No & $100 \%$ & $20 \%$ & $11 \%$ & 116 \\
\hline $500 \mathrm{slpm}$ & $0 \%$ & $0 \%$ & $21 \%$ & 116 \\
$500 \mathrm{slpm}$ & $50 \%$ & $23 \%$ & $11 \%$ & 81 \\
$500 \mathrm{slpm}$ & $100 \%$ & $44 \%$ & $2 \%$ & 64 \\
\hline $1000 \mathrm{slpm}$ & $0 \%$ & $0 \%$ & $27 \%$ & 73 \\
$1000 \mathrm{slpm}$ & $50 \%$ & $10 \%$ & $22 \%$ & 71 \\
$1000 \mathrm{slpm}$ & $100 \%$ & $20 \%$ & $17 \%$ & 67 \\
\hline
\end{tabular}


Analysing the results of Table 4, it can be seen that for the case without quenching a process yield of $11 \%$ and a particle mean diameter of $116 \mathrm{~nm}$ are obtained. In this case, since there is no gas flow through the porous wall section, all the nanoparticles impinging on such wall are assumed to deposit onto it (i.e. $100 \%$ sticking coefficient). In the other cases the simulations were carried out varying the sticking coefficients (using values of $0 \%, 50 \%$ and $100 \%$ ), in order to evaluate its effect on the process performance. The total radial nanoparticle flux towards the walls, including both the diffusive and convective nanoparticle flux, is shown in Figure 5, for the cases at $0 \mathrm{slpm}, 500 \mathrm{slpm}$ and $1000 \mathrm{slpm}$ quench gas flow rate, simulated using a 100\% sticking coefficient. The nanoparticle radial flux in proximity of the walls is considerably higher in the case at $500 \mathrm{slpm}$ compared to the other two cases, resulting also in a higher nanoparticle deposition on the porous wall. A comparison between the two cases with quench gas injection is offered in Figure 6, including both contributions to the radial nanoparticle flux, diffusive and convective fluxes. The convective flux (Figure 6a) is directed inwards in the porous quench wall region, hence towards the negative radial coordinate, therefore the maximum of this flux corresponds to the lowest negative value in the blue colorbar). The diffusive flux (Figure $6 \mathrm{~b}$ ) is directed towards the positive radial coordinate, hence its maximum corresponds to the highest positive value in the red colorbar. 


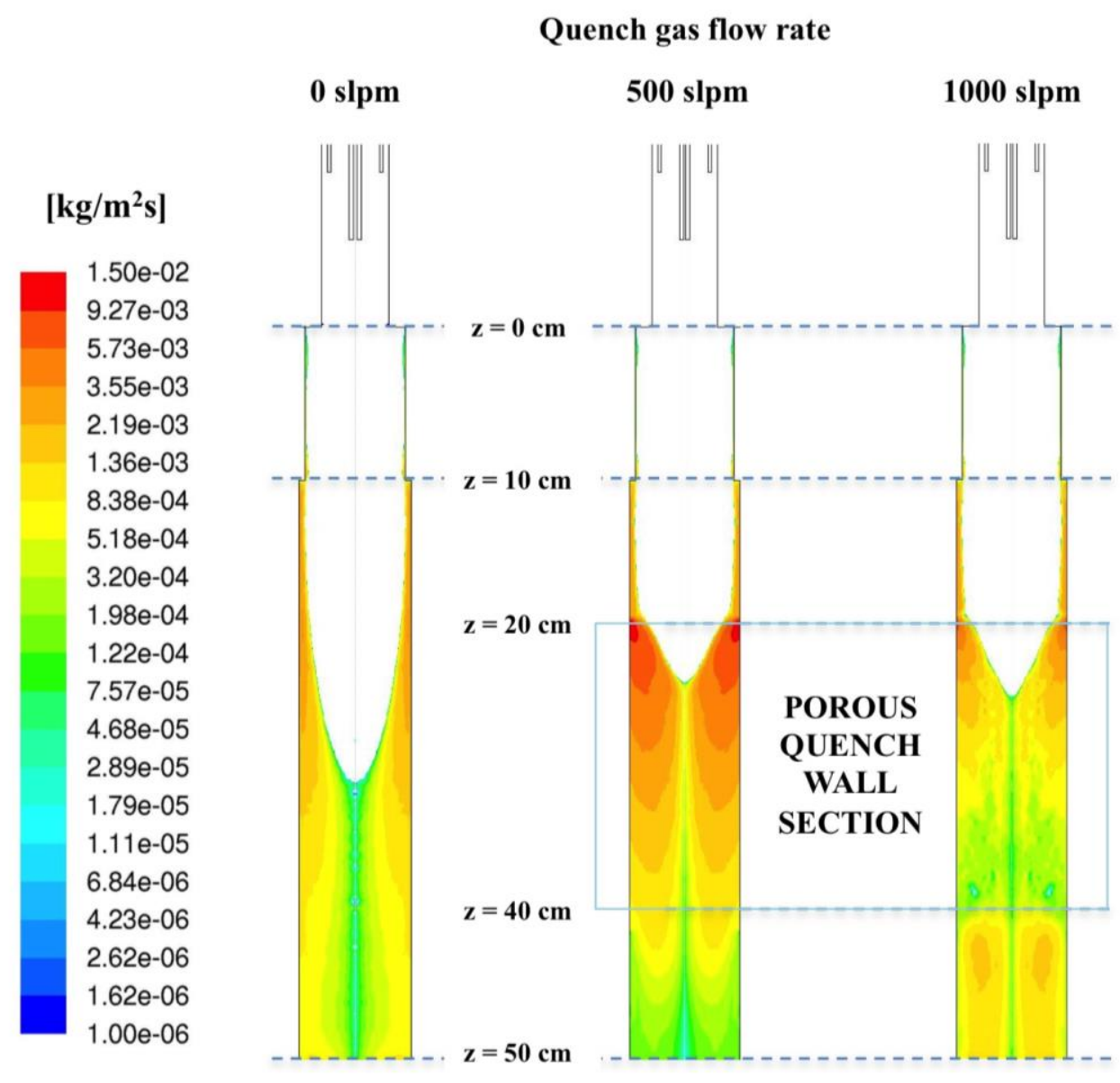

Total nanoparticle radial flux

Figure 5 Nanoparticle radial diffusive flux towards the walls for the cases of no quench gas, 500 slpm and 1000 slpm quench gas flow rate. Simulations were carried out for the case of $60 \mathrm{~kW}$ plate power $(39 \mathrm{~kW}$ coupled power) and $0.46 \mathrm{~g} / \mathrm{s}$ precursor feed rate. 


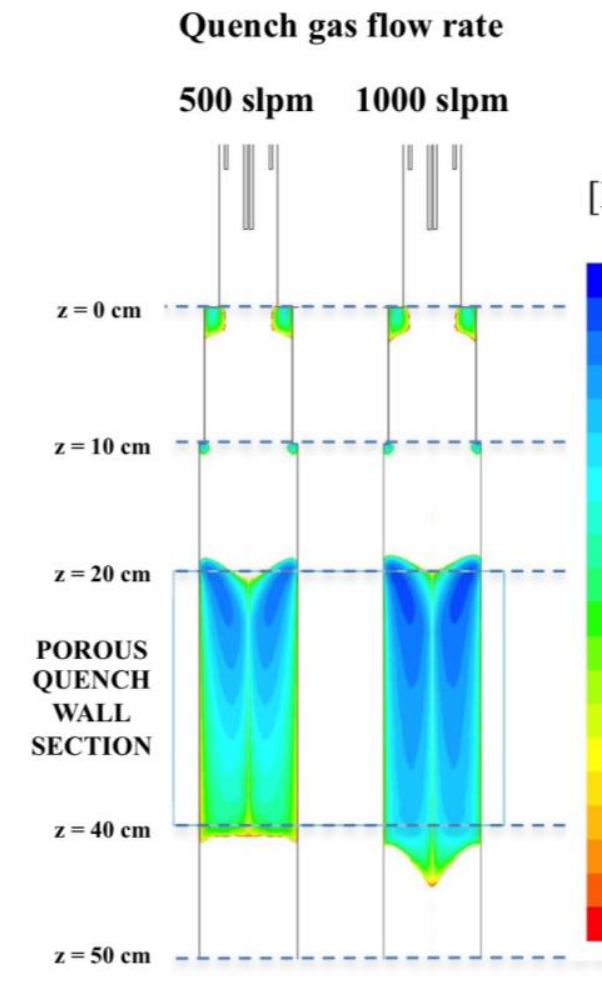

a) Nanoparticle convective flux
Quench gas flow rate

500 slpm 1000 slpm

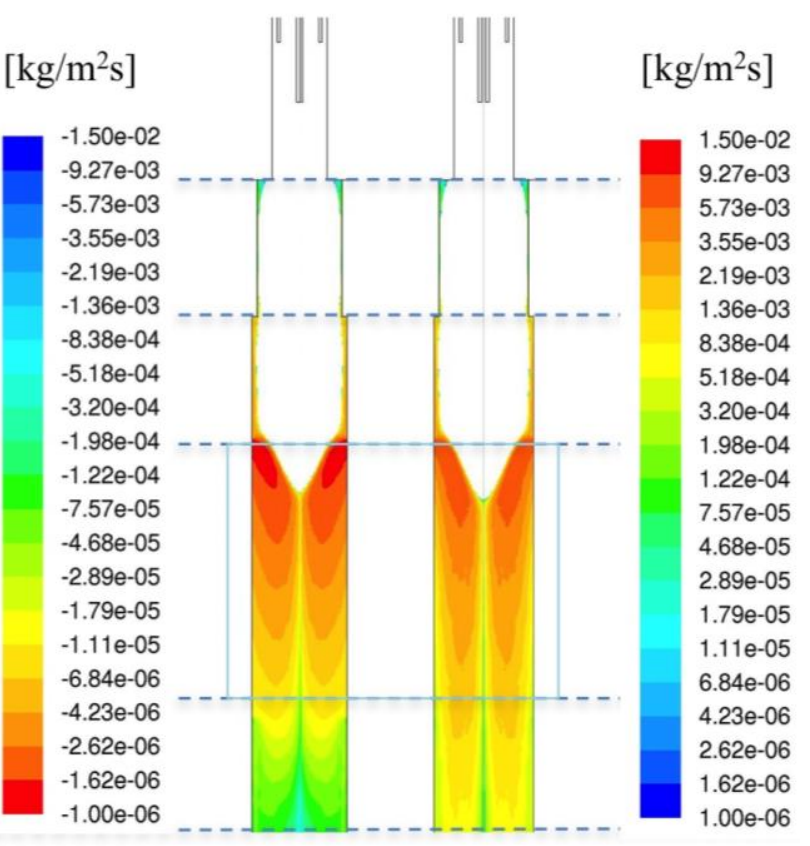

b) Nanoparticle diffusive flux

Figure 6 a) Nanoparticle convective flux in the inward radial direction for the cases at 500 slpm and 1000 slpm quench gas flow rate b) Nanoparticle radial diffusive flux towards the walls for the cases at 500 slpm and 1000 slpm of quench gas flow rate. All simulations were carried out for the case of $60 \mathrm{~kW}$ plate power $(39 \mathrm{~kW}$ coupled power) and $0.46 \mathrm{~g} / \mathrm{s}$ precursor feed rate.

The case at 500 slpm of quench gas flow rate is characterized by both a higher diffusive flux towards the walls, compared to the case at 1000 slpm (Figure 6b), and a lower convective flux of nanoparticles away from the walls (Figure 6a). The latter is due to a lower flow rate of gas entering the reaction chamber through the porous wall, while the former is related to a higher particle diffusivity. The total nanoparticle diffusion coefficient is defined by two contributions:

$$
D_{k}^{\text {tot }}=D_{k}^{L}+D_{P}^{T}
$$

where the index $k=0,1,2$ refers to the moments of the PSD function, $D_{k}^{L}$ is the laminar diffusion coefficient, as usually adopted in the classical moment method [10], and $D_{k}^{T}$ is the particle turbulent diffusion coefficient, computed as [6]:

$$
D_{P}^{T}=\frac{\mu_{T}}{\rho S c}
$$

The particle turbulent diffusion coefficient is reported in Figure 7 for the two cases at 500 slpm and $1000 \mathrm{slpm}$ of quench gas flow rate. A higher turbulent diffusion coefficient is reported for the case at 
500 slpm compared to that at $1000 \mathrm{slpm}$, thus explaining the higher nanoparticle diffusive flux displayed in Figure 6b. The high deposition flux of nanoparticles on the porous wall section in the case at $500 \mathrm{slpm}$ quench gas flow rate is thus due to the combined effect of a higher turbulent diffusion coefficient and of a lower radial inward gas flow.

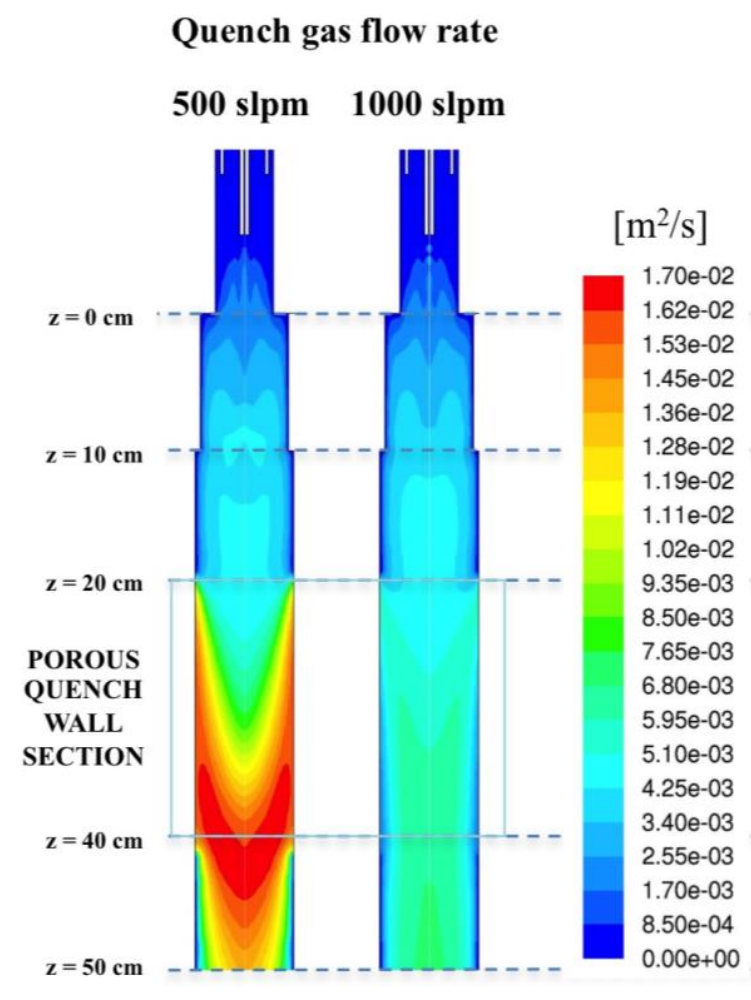

Particle turbulent diffusion coefficient

Figure 7 Particle turbulent diffusion coefficient for the cases at $500 \mathrm{slpm}$ and $1000 \mathrm{slpm}$ of quench gas flow rate. Simulations were carried out for the case of $60 \mathrm{~kW}$ plate power $(39 \mathrm{~kW}$ coupled power) and $0.46 \mathrm{~g} / \mathrm{s}$ precursor feed rate.

Figure 8a reports the profiles of the nanoparticle deposition flux along the porous wall section for the three cases of no quench gas, $500 \mathrm{slpm}$ and $1000 \mathrm{slpm}$ quench gas flow rates, simulated using 100\% sticking coefficient. The particle deposition profile decreases linearly along the porous wall section in the case without quench gas injection, while it is peaked near the top of the porous section in the cases with quench gas injection. This peak is probably related to the combined effect of a high diffusivity (see Figure 7) and a high concentration of nanoparticles (see Figure 9). Since the particle deposition on the porous wall section accounts for a considerable fraction of the nanoparticle losses (44\% in the case at $500 \mathrm{slpm}$ quench gas flow rate), the value of the sticking coefficient assumed on said wall strongly affects the yield of the process, as reported in Table 2. Figure $8 \mathrm{~b}$ shows the particle deposition profiles along the porous wall section for the case at $1000 \mathrm{slpm}$ quench gas flow rate, using three different sticking coefficients $(100 \%, 50 \%$ and $0 \%)$. The lower the sticking coefficient is, the lower the nanoparticle loss due to deposition on the porous wall, resulting in a higher process yield. A maximum yield of $27 \%$ was obtained for the case at 1000 slpm and $0 \%$ sticking coefficient (see Table 2). 
Furthermore, lower sticking coefficients result in higher mean diameters of the nanoparticles at the outlet of the chamber, as particle growth mechanisms (coagulation and coalescence) are enhanced due to the higher concentration of particles downstream the quench wall section.
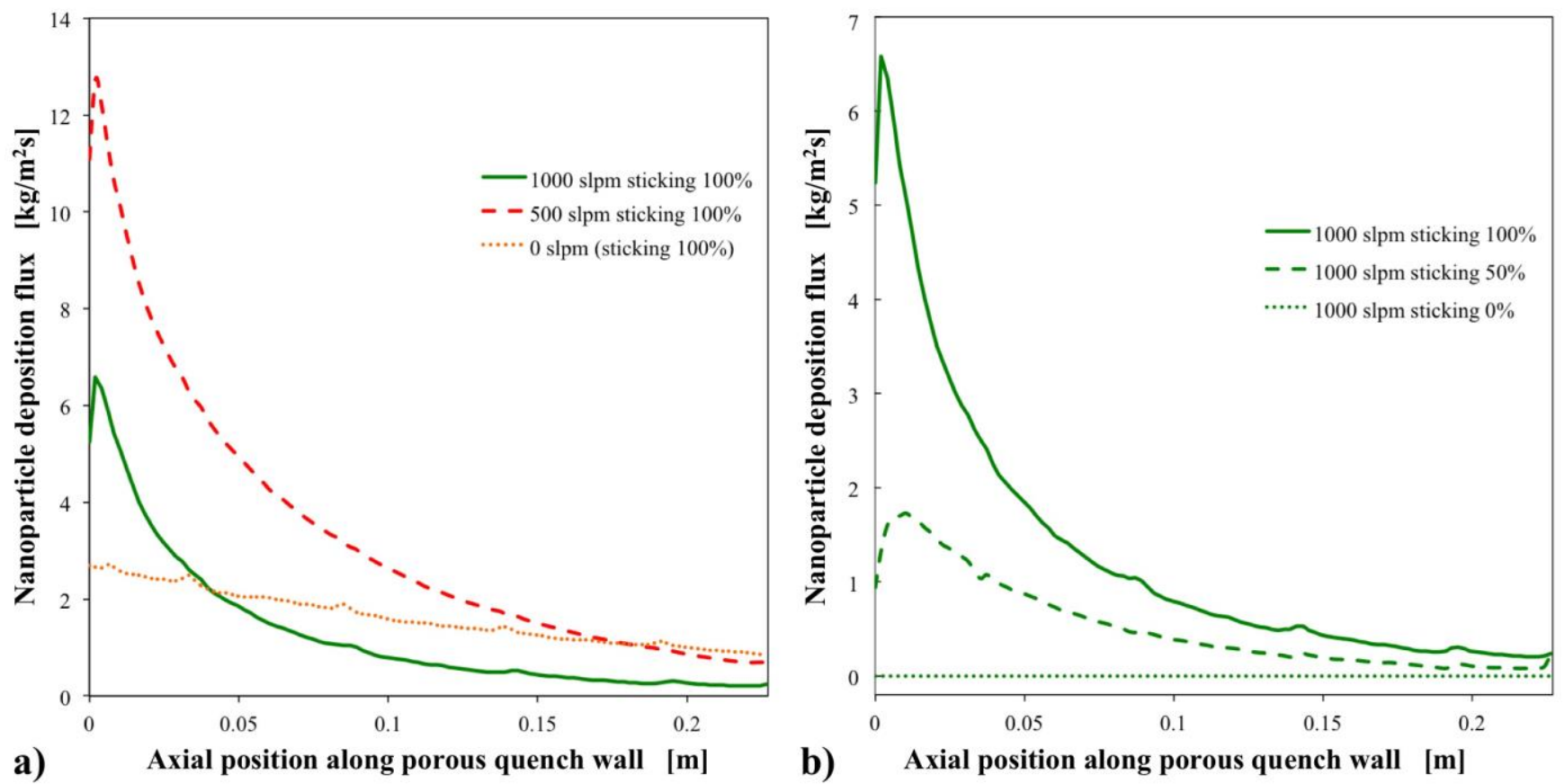

Figure 8 a) Nanoparticle deposition flux on the porous quench wall section as a function of quench gas flow rate, assuming a $100 \%$ sticking coefficient b) Nanoparticle deposition flux on the porous quench wall section as a function of sticking coefficient. All the simulations were carried out for the case of $60 \mathrm{~kW}$ plate power $(39 \mathrm{~kW}$ coupled power) and $0.46 \mathrm{~g} / \mathrm{s}$ precursor feed rate.

Figure 9 shows a comparison of the nanoparticle concentration in the chamber for the cases at 1000 slpm quench gas flow rate simulated using three different sticking coefficients $(100 \%, 50 \%$ and $0 \%)$. All three cases are characterized by a high particle diffusion flux towards the wall in the top section of the porous wall, as shown in Figure $6 \mathrm{~b}$ and $7 \mathrm{~b}$. The nanoparticle concentration in proximity of the porous wall section increases with a decrease in the sticking coefficient as a result of the nanoparticle flux that is partially or totally reflected into the chamber by the inward quenching gas flow. 


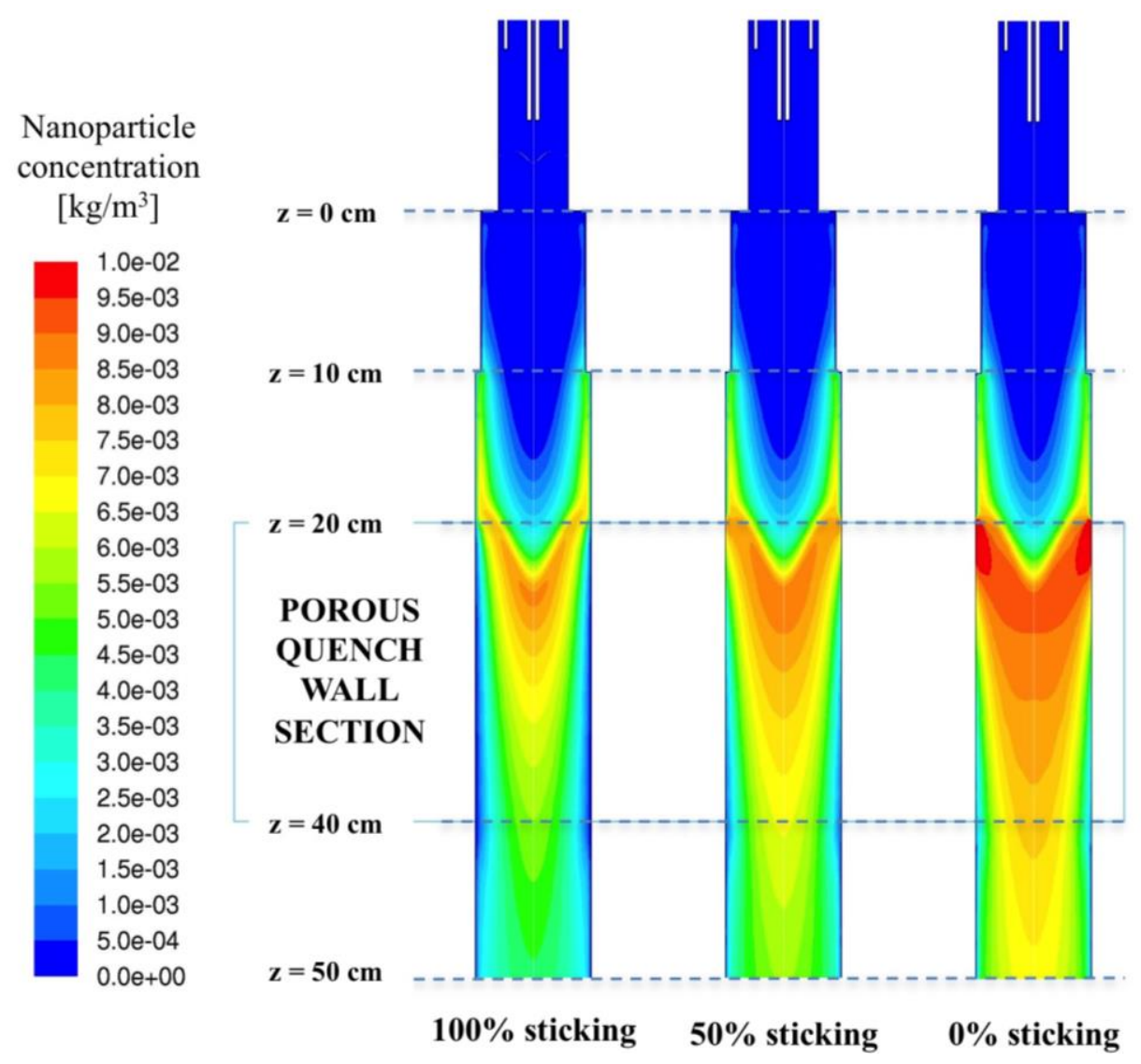

Figure 9 Nanoparticle concentration profile in the cylindrical reaction chamber for the cases at 1000 slpm quench gas flow rate, simulated using sticking coefficients of $100 \%, 50 \%$ and $0 \%$. Simulations were carried out for the case of $60 \mathrm{~kW}$ plate power $(39 \mathrm{~kW}$ coupled power) and $0.46 \mathrm{~g} / \mathrm{s}$ precursor feed rate.

Nanoparticle synthesis with a conical chamber

The use of a reaction chamber with a conical top region has been investigated by Pristavita et al. [30] and by Colombo et al. [13], concluding that a finer control of the flow pattern (loss of nanoparticles to the walls) and nanoparticle properties (size) can be achieved thanks to its shape with respect to the cylindrical case. Furthermore, it was shown that the injection of a shroud gas in synergy with the chamber geometry allows to confine the nucleation region away from the walls and to reduce the nanoparticle deposition on them, resulting in higher process yield [13].

Table 5 reports the yield and mean diameter of synthetized nanoparticle for the case of a conical reaction chamber with $500 \mathrm{slpm}$ of shroud gas $(250 \mathrm{slpm}$ for each of the two inlet regions, as shown in Figure 1), compared to the corresponding case in the cylindrical chamber, with $500 \mathrm{slpm}$ quench gas flow rate and 50\% sticking coefficient on the porous wall section. The synergic effect of the geometry and shroud gas in the conical chamber allows achieving both a high process yield (48\%) and a low 
particle diameter $(77 \mathrm{~nm})$. Conversely, the quench gas injection in the cylindrical chamber does not positively affect the process yield, due to the turbulent diffusion phenomena discussed in the previous section. The nanoparticle concentration (displayed in Figure 10a) is peaked on the porous wall for the case of the cylindrical chamber, while it is peaked on the axis and low in proximity of the walls for the case of the conical chamber, thanks to the effect of the shroud gas flow. Similarly, the diffusive flux towards the walls (shown in Figure 10b) is lower in the conical chamber compared to the cylindrical chamber. A higher nanoparticle yield is thus achieved in the case of a conical chamber with $500 \mathrm{slpm}$ shroud gas flow rate, as a result of a lower particle loss through deposition on the chamber walls. The nanoparticle mass-weighted mean diameter through the two chambers is shown in Figure 11: the reduced length of the conical chamber results in a lower nanoparticle size at the outlet, due to a lower residence time of the synthetized nanoparticles.

Table 5: Summary of the yield and mean diameter of the synthetized nanoparticles in the two chambers (cylindrical and conical) for the both the case without quench gas and with $500 \mathrm{slpm}$ quench gas flow rate. The quench gas is delivered through two inlets in the conical chamber $(250 \mathrm{slpm}$ from each), while the porous quench wall in the cylindrical chamber was modelled using a 50\% sticking coefficient. All simulations were carried out for $60 \mathrm{~kW}$ plate power $(39 \mathrm{~kW}$ coupled power) and a precursor feed rate of $0.46 \mathrm{~g} / \mathrm{s}$.

\begin{tabular}{llll}
\hline Chamber & Quench & $\begin{array}{l}\text { Yield } \\
(\%)\end{array}$ & $\begin{array}{c}\overline{\boldsymbol{d}}_{\boldsymbol{P}} \text { at outlet } \\
(\mathrm{nm})\end{array}$ \\
\hline \multirow{2}{*}{ Cylindrical } & No & $11 \%$ & 116 \\
& 500 slpm & $11 \%$ & 81 \\
\hline \multirow{2}{*}{ Conical } & No & $16 \%$ & 87 \\
& 500 slpm & $48 \%$ & 77 \\
\hline
\end{tabular}




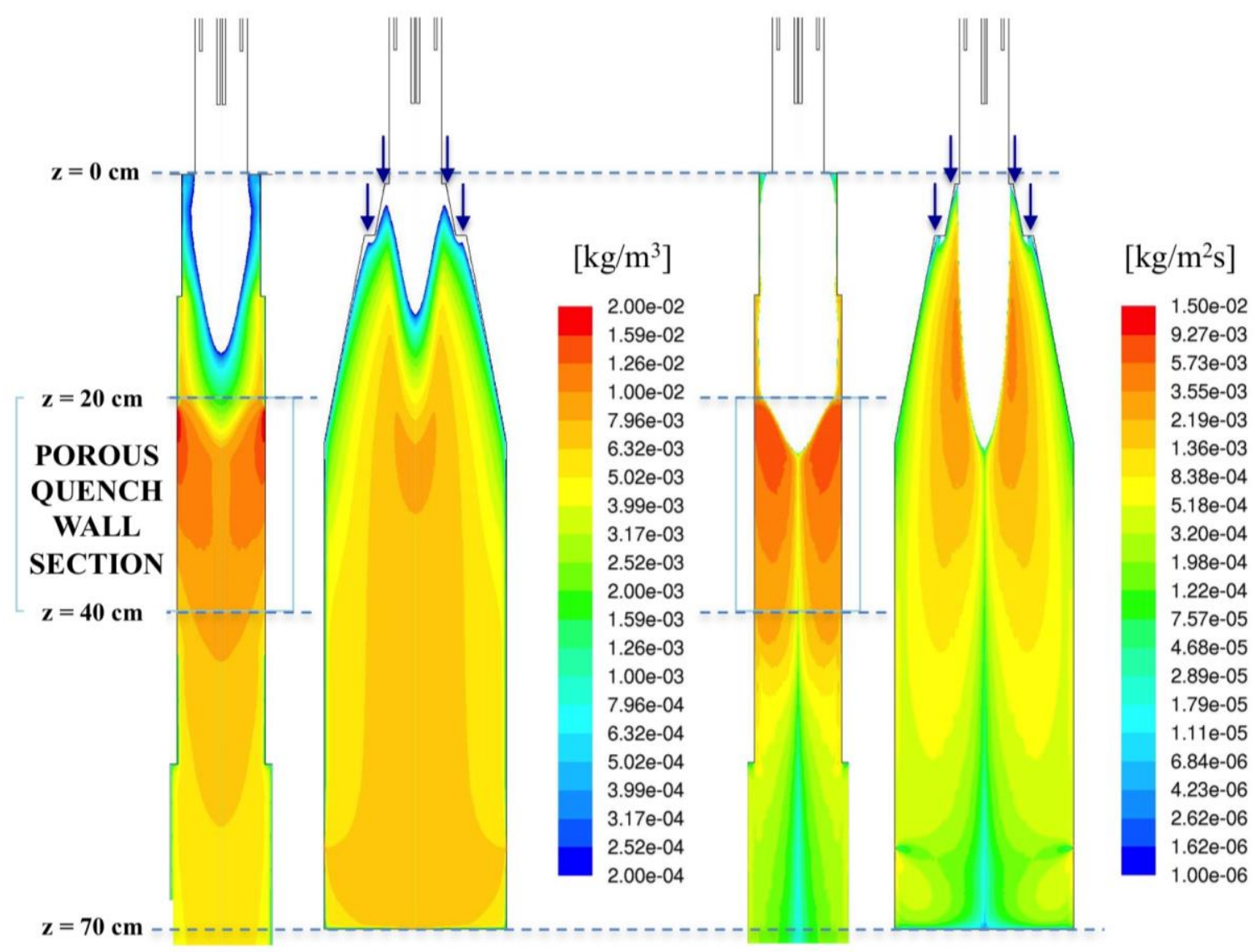

a) Nanoparticle concentration

b) Nanoparticle diffusive flux

Figure 10 a) Nanoparticle concentration for the cases of the cylindrical chamber (left) and the conical chamber (right) b) Nanoparticle radial diffusive flux towards the walls for the cases of the cylindrical chamber (left) and the conical chamber (right) All simulations were carried out for the case of $60 \mathrm{~kW}$ plate power (39 $\mathrm{kW}$ coupled power), $500 \mathrm{slpm}$ of quench gas flow rate and $0.46 \mathrm{~g} / \mathrm{s}$ precursor feed rate. 


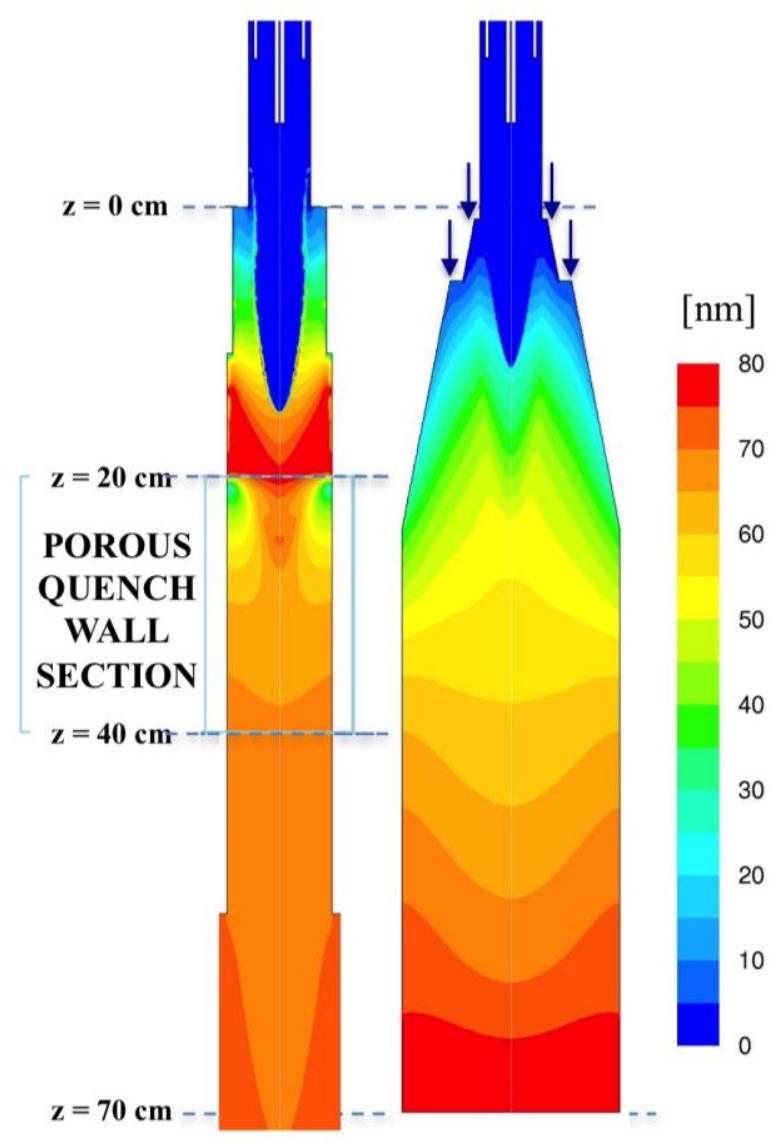

Nanoparticle mean diameter

Figure 11 Mean diameter of the synthetized nanoparticles for the cases of the cylindrical chamber (left) and the conical chamber (right). All simulations were carried out for the case of $60 \mathrm{~kW}$ plate power $(39 \mathrm{~kW}$ coupled power), $500 \mathrm{slpm}$ of quench gas flow rate and $0.46 \mathrm{~g} / \mathrm{s}$ precursor feed rate.

Stream functions inside each reaction chamber are reported in Figure 12. In the cylindrical chamber vortices are generated close to the outlet of the plasma torch and below the porous quench wall section. These vortices affect PSD by increasing the particle mean size and have negative effect on the yield, as they increase the residence time of the powders in the chamber and the amount of powders lost to the walls. On the other hand, the conical chamber presents a laminar behavior with no notable vortices. Velocity field vectors are reported in Figure 13 for each reaction chamber, with a close up on the plasma torch exit region and on the conical chamber shroud gas inlets one. The injection velocity of the quench gas coming from the porous wall is considerably lower than the shroud gas one, even with the same flow rate. In this paper the shroud were considered to be axially injected, but there is no technical limitations to have multiple injections point with tilted inlet direction and use this additional degree of freedom for process optimization in terms of yield and size distribution. 


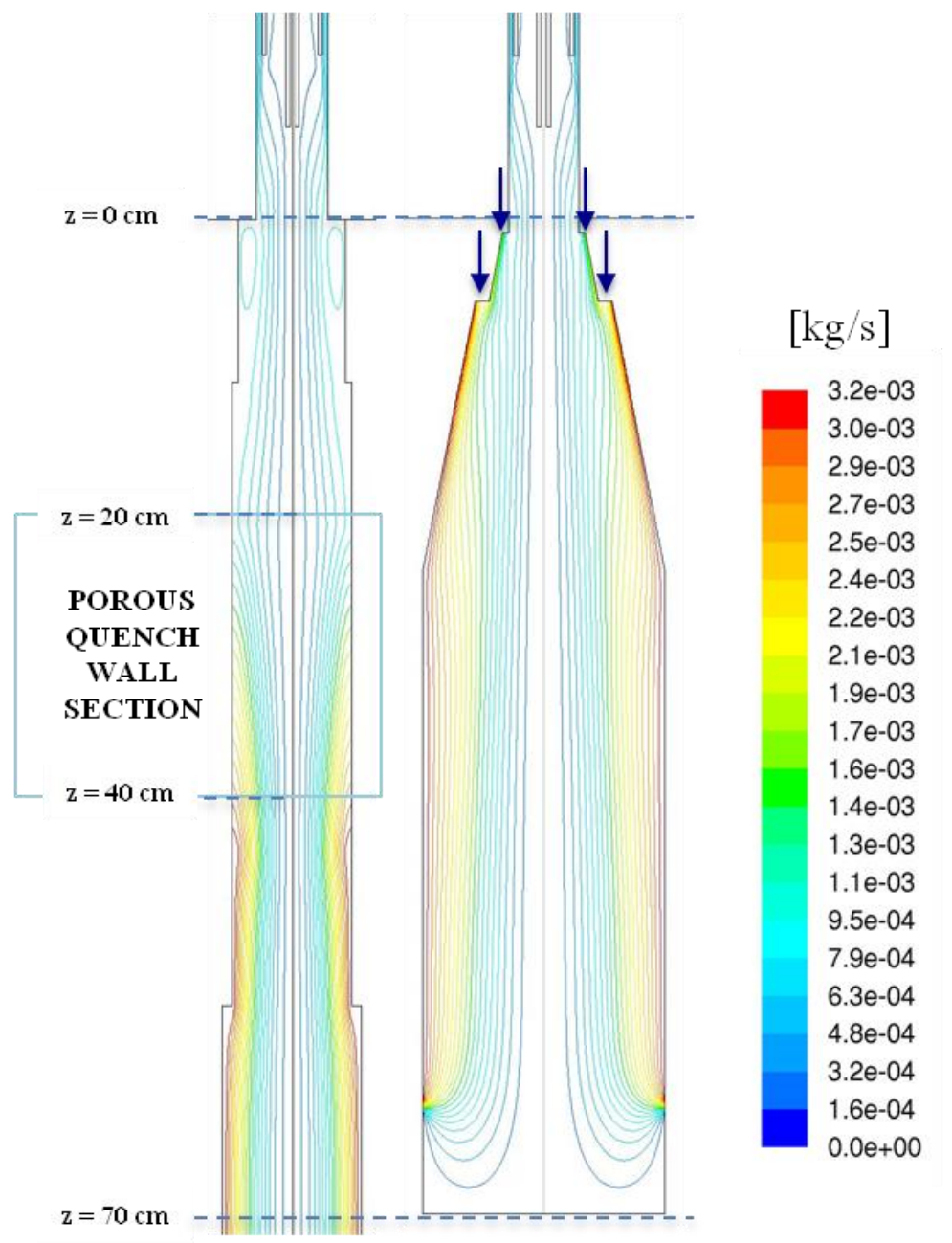

Figure 12 Stream function for the cases of the cylindrical chamber (left) and the conical chamber (right). All simulations were carried out for the case of $60 \mathrm{~kW}$ plate power ( $39 \mathrm{~kW}$ coupled power), $500 \mathrm{slpm}$ of quench gas flow rate and $0.46 \mathrm{~g} / \mathrm{s}$ precursor feed rate. 


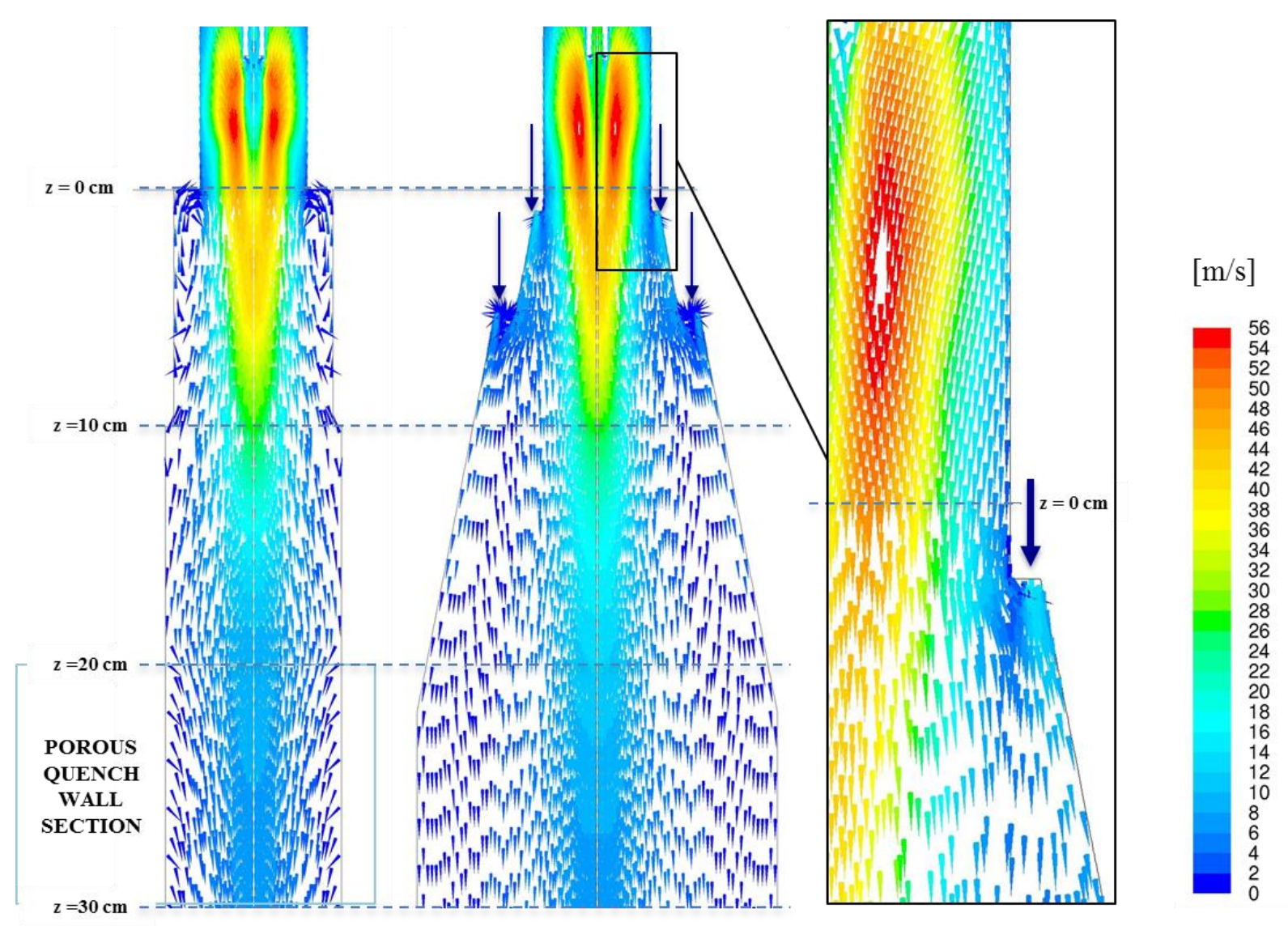

Figure 13. Vector velocity fields for the cases of the cylindrical chamber (left) and the conical chamber (center, right). All simulations were carried out for the case of $60 \mathrm{~kW}$ plate power ( $39 \mathrm{~kW}$ coupled power), $500 \mathrm{slpm}$ of quench gas flow rate and $0.46 \mathrm{~g} / \mathrm{s}$ precursor feed rate.

\section{Conclusions}

The performance of the copper nanoparticle synthesis process in a RF-ITP is investigated using a thermo-fluid dynamic model combined with electromagnetic field equations, a precursor tracking model and a moment model for nanoparticle synthesis and transport. When the radiation losses from the $\mathrm{Cu}$ vapour are correctly included in the model, a significant reduction of the temperature is observed in the region downstream the injection probe, resulting in a lower evaporation efficiency for the injected solid micrometric precursor. A new method to model the nanoparticle deposition onto a porous wall carrying a gas flow into the reaction chamber is proposed, and the effects of varying the sticking coefficient of the particles on the walls are highlighted. Finally, a comparison between two different chamber designs and quenching strategies is offered, with regards to the size distribution and yield of the synthetized nanoparticles. The design-oriented modelling approach adopted in the present work allowed to gain insights on some of the phenomena governing the nanoparticle synthesis process, providing guidance towards the optimization and upscaling of the process, as an alternative to expensive try and fail experimental approaches. The adoption of a porous quench wall solution, which can be considered as an "active" quenching, allows controlling efficiently the nanoparticle mean diameter, but with low production yield. A shroud gas, which instead can be considered as a "passive" 
quenching, has less effect in controlling the nanoparticle mean diameter but can reach very high nanoparticle yield, which is an important industrial target. Although the optimization of the process parameters for the nanoparticle synthesis in a RF-ITP system is highly dependent on the properties of the material to be synthetized, the results shown in the present work can nonetheless be extended to different materials and operating conditions. The model can still be improved by including other physical phenomena, such as thermophoresis: for example, investigating the sensitivity of the synthesis process to thermophoretic effects for different quenching strategies [31]. Also the design of a conical reaction chamber can be further improved by a research parameterized on different injection modality for the shroud gas [13]. Simulations results also provide insights for the thermo-fluid mechanic design of reaction chambers, especially in the more thermally stressed regions of the chamber. As it is possible that some of the nanoparticles that reach the water cooled walls will not stick onto them, future work could be aimed to investigate the impact on process yield of hypothesizing different sticking coefficients also for these walls.

\section{Acknowledgements}

The authors would like to thank Dr. Emanuele Ghedini for some helpful discussion concerning this work. Financial support from the European Union within the Horizon 2020 research and innovation programme, under grant agreement n. 646155 (INSPIRED project http://www.nano-inspired.eu/), is gratefully acknowledged.

This is a post-peer-review, pre-copyedit version of an article published in Plasma Chemistry and Plasma Processing.

The final authenticated version is available online at: http://doi.org/10.1007/s11090-016-9779-1

Published under the

"Springer Nature terms of reuse for archived author accepted manuscripts (AAMs) of subscription articles": http://www.springer.com/gp/open-access/authors-rights/aam-terms-v1 


\section{References:}

[1] Ostrikov K and Murphy A B 2007 J. Phys. D: Appl. Phys. 40 2223-2241

[2] Ostrikov K, Cvelbar U and Murphy A B 2011 J. Phys. D: Appl. Phys. 44174001

[3] Shigeta M and Murphy A B 2011 J. Phys. D: Appl. Phys. 44174025

[4] Seo J H and Hong B G 2012 Nucl. Eng. Tech. 44 9-20

[5] Murphy A B, Boulos M I, Colombo V, Fauchais P, Ghedini E, Gleizes A, Mostaghimi J, Proulx P and Schram D C 2008 High Temp. Mater. Process. 12 255-336

[6] Colombo V, Ghedini E, Gherardi M, Sanibondi P and Shigeta M 2012 Plasma Sci. Technol 21025001

[7] Shigeta M and Nishiyama H 2005 J. Heat Transfer 127 1223-1231

[8] Colombo V, Ghedini E, Gherardi M and Sanibondi P 2013 Plasma Sources Sci. Technol. 22 035010

[9] Boulos M I 2015 Plasma Chem. Plasma Process. DOI 10.1007/s11090-015-9660-7

[10] Shigeta M and Watanabe T 2008 J. Appl. Phys. 103074903

[11] Mendoza-Gonzalez N Y, Goortani B M and Proulx P 2007 Material Science and Engineering C 27 1265-1269

[12] Goortani B M, Mendoza-Gonzalez N Y and Proulx P 2006 Int. J. Chem. Reactor Eng. 4 A33

[13] Colombo V, Ghedini E, Gherardi M and Sanibondi P 2012 Plasma Sci. Technol 21055007

[14] Goortani B M, Proulx P, Xue S and Mendoza-Gonzalez N Y 2007 Powder Technol. 175 2232

[15] ANSYS 2013 ANSYS FLUENT 15.0 Theory Guide (Canonburg, PA)

[16] Bilodeau J F and Proulx P 1996 Aerosol Sci. Technol. 24 175-189

[17] Cressault Y and Gleizes A 2013 J. Phys. D: Appl. Phys. 46415206

[18] Gleizes A, Cressault Y and Teulet P 2010 Plasma Sci. Technol. 19055013

[19] Colombo V, Ghedini E and Sanibondi P 2010 Plasma Sci. Technol 19065024

[20] Mendoza-Gonzalez N Y, Morsli M E and Proulx P 2008 J. Therm. Spray Technol. 17 533550

[21] Friedlander S K 2000 Smoke, Dust and Haze, Fundamentals of Aerosol Dynamics $2^{\text {nd }}$ edn (Oxford: Oxford University Press)

[22] Fuchs N A 1964 Mechanics of Aerosols (New York: Pergamon)

[23] Phanse G M and Pratsinis S E 1989 Aerosol Sci. Technol. 11 100-119

[24] Pratsinis S E 1988 J. Coll. Interf. Sci. 124 416-427

[25] Bernardi D, Colombo V, Ghedini E and Mentrelli A 2003 Eur. Phys. J. D 27 55-72

[26] Chen K and Boulos M I 1994 J. Phys. D: Appl. Phys. 27946

[27] Colombo V, Ghedini E and Sanibondi P 2008 Progr. Nucl. Energy 50921

[28] Bourasseau E, Homman A, Durand O, Ghoufi A and Malfreyt P 2013 Eur. J Phys. B 86251

[29] Lide D R 2003 Handbook of Chemistry and Physics, 84 ${ }^{\text {th }}$ Ed. (CRC Press)

[30] Pristavita R, Mendoza-Gonzales N Y, Meunier J L and Berk D 2010 Plasma Chem. Plasma Process. 30 267-79

[31] Bianconi S, Boselli M, Gherardi M and Colombo V 2016 submit. to J. Phys. D: Appl. Phys. 